\title{
Stochastic Scheduling Problems for Maximizing the Expected Number of Early Jobs with Common or Exchangeable Due Dates
}

\author{
Jae Young Choi \\ R\&D Center, Dongbu CNI \\ Heung-Kyu Kim* \\ School of Business, Dankook University
}

(Received: May 5, 2012 / Revised: May 20, 2012 / Accepted: May 30, 2012)

\begin{abstract}
In this paper, stochastic scheduling problems are considered when processing times and due dates follow arbitrary distributions and due dates are either common or exchangeable. For maximizing the expected number of early jobs, two policies, one, based on pairwise comparisons of the processing times, and the other, based on survivabilities, are introduced. In addition, it is shown that the former guarantees optimal solutions when the processing times and due dates are deterministic and that the latter guarantees optimal solutions when the due dates follow exponential distributions. Then a new approach, exploiting the two policies, is proposed and analyzed which turns out to give better job sequences in many situations. In fact, the new approach guarantees optimal solutions both when the processing times and due dates are deterministic and when the due dates follow exponential distributions.
\end{abstract}

Keywords: Stochastic Scheduling Problem, Early Job, Common Due Date, Exchangeable Due Dates

* Corresponding Author, E-mail: heungkyu@dankook.ac.kr

\section{INTRODUCTION}

The problem of maximizing the expected number of early jobs with a common due date ${ }^{1)}$ is usually applied to the situation where random breakdown of a machine is considered with the time of breakdown being the common due date. The number of job completions should be maximized before the machine breaks down.

In addition, the problem of maximizing the expected number of early jobs with exchangeable due dates ${ }^{2)}$ frequently appears in agriculture, especially when perishable goods are involved (Sterna, 2006). For instance, crops

1) The same due date is assigned to all the jobs remaining to be processed.

2) The same distribution of due date is assigned to all the jobs remaining to be processed. being collected from different stretches of land should be harvested. Crops perish if not collected prior to exchangeable due dates, determined by the mixture of land attributes and nature. Therefore maximizing the expected number of early jobs are the same as minimizing the amount of wasted crops.

The problems of maximizing the expected number of early jobs with a common due date or exchangeable due dates have been investigated in many literatures (Choi and Kim, 2011).

For instance, it is assumed that the processing times follow normal distributions with variances proportional to their respective processing time means and the common due date is deterministic. It is found that the optimal static policies are $\mathrm{V}$ or $\mathrm{W}$-shaped where the shapes are based on their respective processing time means (Sarin et al., 1991). Similar problems without assuming 
proportional variances are studied, where 'normalized slack policy' is introduced which is optimal for both static $^{3)}$ and dynamic ${ }^{4)}$ scheduling in some cases (Jang, 2002).

Problems with arbitrary processing times and an exponential due date are considered. It is shown that if processing times are NBU (new-better-than-used), ${ }^{5)}$ the optimal static policy is preserved in the optimal dynamic policy as well (De et al., 1991). Similar problems with a more general objective function which includes earliness penalties are considered (Cai et al., 1999). In addition, stochastic scheduling problems with general distributional assumptions on processing times are investigated (Li et al., 1998).

In this paper, the problem of maximizing the expected number of early jobs through a single machine is considered. There are $n$ jobs to be processed with processing times $X_{i}, i=1,2, \cdots, n$. It is assumed that jobs are not resumed once the due date occurs and that all the remaining unprocessed jobs cannot be processed thereafter. The due date for each job $i, D_{i}, i=1,2, \cdots, n$, is common $(=D)$ or exchangeable. Both processing times and due dates are assumed to follow arbitrary distributions. This paper aims to find a static policy of getting a sequence $\pi\left(j_{1}, j_{2}, \cdots, j_{n}\right)$ of $n$ jobs in such a way that the expected number of early jobs $E[\pi]$ is maximized.

The rest of this paper is organized as follows. Two policies are introduced in Section 2. One of them is based on pairwise comparisons of processing times, and the other is based on survivabilities. Then, in Section 3, a new policy, exploiting the two policies, is derived and analyzed, which gives better job sequences than the two policies in many situations. In conclusion, the proposed policy turns out to capture advantages of both policies, which is summarized in Table 1. Final remarks are addressed in Section 4.

Table 1. Comparison

\begin{tabular}{|c|c|c|c|c|}
\hline $\begin{array}{c}\text { Processing } \\
\text { Times }\end{array}$ & Due Date & $\begin{array}{c}\text { Pairwise } \\
\text { Rank } \\
\text { Based } \\
\text { Policy }\end{array}$ & $\begin{array}{c}\text { Probabilistic } \\
\text { Slack Policy }\end{array}$ & $\begin{array}{c}\text { Proposed } \\
\text { Policy }\end{array}$ \\
\hline Any & Exponential & $\begin{array}{c}\text { Not } \\
\text { optimal }\end{array}$ & Optimal & Optimal \\
\hline Exponential & Any & Optimal & Optimal & Optimal \\
\hline $\begin{array}{c}\text { Deterministic } \\
\text { Deterministic }\end{array}$ & Optimal & Not optimal & Optimal \\
\hline $\begin{array}{c}\text { All distributions are } \\
\text { non-overlapping }\end{array}$ & Optimal & Not optimal & Optimal \\
\hline
\end{tabular}

3) A set of jobs are scheduled before any one of them is processed, and once the first job is being processed, the sequence of the remaining jobs is never revisited until all the jobs are finished.

4) The order of jobs to be processed can be revisited at any time, utilizing all the information available up to that point in time.

5) The probability that a new unit will survive time $t$ is greater than or equal to the probability that a used unit of age $S$ will survive an additional time $t$.

\section{ALGORITHMS}

In this section, 'pairwise rank based policy' is introduced which is obtained from a sample path analysis. It is shown that the policy is optimal when the processing times are ordered in failure rate sense. Then 'probabilistic slack policy' is introduced as well. It is shown that the policy is optimal when due date is exponential. Finally, a new policy is proposed to exploit the two policies.

\subsection{Pairwise Rank Based Policy}

\subsubsection{Stochastic Ordering}

The probability that $t$ is between the processing time of job $i, X_{i}$, and the sum of processing times of jobs $i$ and $j, X_{i}+X_{j}$, i.e., $g_{t}(i, j)=\operatorname{Pr}\left\{X_{i} \leq t<X_{i}+X_{j}\right\}$, upon which optimal policy is based, is introduced. It is shown that if $g_{t}(i, j) \geq g_{t}(j, i)$ holds for all $t \geq 0$, then sequence $\pi\left(\pi_{1}, i, j, \pi_{2}\right)$ is better than sequence $\pi^{\prime}\left(\pi_{1}\right.$, $\left.j, i, \pi_{2}\right)$, where the two adjacent jobs $i$ and $j$ in sequence $\pi\left(\pi_{1}, i, j, \pi_{2}\right)$ are interchanged (Li et al., 1998). Even though it seems that this sufficient condition is quite general, it turns out that the condition leads to a stochastic ordering. That is, if we denote the distribution function and probability density function of $X_{i}$ by $F_{i}$ and $f_{i}$, respectively, and $\bar{F}_{i}=1-F_{i}$, then

$$
\begin{aligned}
\operatorname{Pr} & \left\{X_{i} \leq t<X_{i}+X_{j}\right\} \\
& =\int_{0}^{\infty} \operatorname{Pr}\left\{X_{i} \leq t<X_{i}+x \mid X_{j}=x\right\} d F_{j}(x) \\
& =\int_{0}^{\infty} F_{i}(t) \bar{F}_{i}(t-x) d F_{j}(x) \\
& =\int_{0}^{t} F_{i}(t) \bar{F}_{i}(t-x) d F_{j}(x)+\int_{t}^{\infty} F_{i}(t) d F_{j}(x) \\
& =F_{i}(t)\left[\int_{0}^{t} \bar{F}_{i}(t-x) d F_{j}(x)+\bar{F}_{j}(t)\right] \\
& =\operatorname{Pr}\left\{X_{i} \leq t\right\} \operatorname{Pr}\left\{X_{i}+X_{j}>t\right\}
\end{aligned}
$$

Therefore, the condition $g_{t}(i, j) \geq g_{t}(j, i)$, for all $t \geq 0$, leads to the following:

$$
\begin{aligned}
& \operatorname{Pr}\left\{X_{i} \leq t<X_{i}+X_{j}\right\} \geq \operatorname{Pr}\left\{X_{j} \leq t<X_{i}+X_{j}\right\} \\
\Leftrightarrow & F_{i}(t) \operatorname{Pr}\left\{X_{i}+X_{j}>t\right\} \geq F_{j}(t) \operatorname{Pr}\left\{X_{i}+X_{j}>t\right\} \\
\Leftrightarrow & F_{i}(t) \geq F_{j}(t)
\end{aligned}
$$

The last inequality is just the stochastic ordering relationship $X_{i} \leq_{s t} X_{j}$ (Ross, 1996).

\subsubsection{More Likely Ordering}

Even though the assumption that processing times are ordered in a stochastic ordering sense is useful, it is not likely to be satisfied. In other words, the data in practice do not come in a stochastic ordering sense. Consequently, a scheduling procedure based on such condi- 
tions often leads to limited partial sequencing. Therefore, weaker ordering conditions are needed. The following lemma does not require the assumption of stochastic ordering. Thus, it can be used for any set of processing times that cannot be ordered in a stochastic ordering sense.

\section{Lemma 1}

Consider any two adjacent jobs $i$ and $j$ with any common due date. If $\operatorname{Pr}\left\{X_{i} \leq X_{j}\right\} \geq \operatorname{Pr}\left\{X_{i}>X_{j}\right\}$, then job $i$ should precede job $j$ in optimal sequence.

\section{Proof}

Consider a realization $\varpi \in \Omega$ of processing times, and denote the realization of $X_{i}$ by $X_{i}(\varpi)$. If $X_{i}(\varpi)<X_{i}$ $(\varpi)$, then it is better to process job $i$ first for any realization of due date. Otherwise, it is better to process job $j$ first. Therefore, in this case, with probability $\operatorname{Pr}\left\{X_{i} \leq\right.$ $\left.X_{j}\right\}$, it is better to process job $i$ first for whatever the realization of due date is.

Denote a case where $X_{i}$ is more likely smaller than $X_{j}$, i.e., $\operatorname{Pr}\left\{X_{i} \leq X_{j}\right\} \geq \operatorname{Pr}\left\{X_{i}>X_{j}\right\}$, by $X_{i} \prec X_{j}$. In general, the relation $X_{i} \prec X_{j}$ is not transitive. In other words, the conjunctive condition that $X_{i} \prec X_{j}$ and $X_{j} \prec X_{k}$ does not always imply $X_{i} \prec X_{k}$. For example, the following three discrete random variables $X, Y$ and $Z$ are not transitive

$$
\left\{\begin{array} { l } 
{ \operatorname { P r } \{ X = 2 \} = 1 / 3 } \\
{ \operatorname { P r } \{ X = 5 \} = 2 / 3 }
\end{array} \quad \left\{\begin{array}{l}
\operatorname{Pr}\{Y=3\}=2 / 3 \\
\operatorname{Pr}\{Y=6\}=1 / 3
\end{array} \quad Z=4\right.\right.
$$

since $X \prec Y$ and $Y \prec Z$, but $Z \prec X$.

However, the transitivity does hold for exponential random variables. Let $X_{i}, X_{j}$ and $X_{k}$ be exponential random variables with respective rates, $\lambda_{i}, \lambda_{j}$ and $\lambda_{k}$. Since $\operatorname{Pr}\left\{X_{i} \leq X_{j}\right\}=\lambda_{i} /\left(\lambda_{i}+\lambda_{j}\right)$, the conjunctive condition that $X_{i} \prec X_{j}$ and $X_{j} \prec X_{k}$, implies that $\lambda_{i} \geq \lambda_{j}$ and $\lambda_{j} \geq \lambda_{k}$ which, in turn, leads to $\lambda_{i} \geq \lambda_{k}$. Thus we have $X_{i} \prec X_{k}$.

\subsubsection{Failure Rate Ordering}

A random variable $X_{i}$ is said to be stochastically smaller than a random variable $X_{j}$ in a failure rate sense if

$$
\frac{f_{i}(t)}{\bar{F}_{i}(t)} \geq \frac{f_{j}(t)}{\bar{F}_{j}(t)} \quad \text { for all } .
$$

This ordering is denoted by $X_{i} \leq_{f} X_{j}$. The following lemma shows that this failure rate ordering implies more likely ordering.

\section{Lemma 2}

If $X_{i} \leq_{f} X_{j}$, then $X_{i} \prec X_{j}$
Proof

$$
\begin{gathered}
X_{i} \leq_{f} X_{j} \\
\Rightarrow \quad \frac{f_{i}(t)}{\overline{F_{i}}(t)} \geq \frac{f_{j}(t)}{\bar{F}_{j}(t)} \quad \text { for all t } \\
\Rightarrow \int_{0}^{\infty} f_{i}(t) \bar{F}_{j}(t) d t \geq \int_{0}^{\infty} f_{j}(t) \bar{F}_{i}(t) d t \\
\Rightarrow \operatorname{Pr}\left\{X_{i} \leq X_{j}\right\} \geq \operatorname{Pr}\left\{X_{i}>X_{j}\right\}
\end{gathered}
$$

The transitivity holds for the random variables that can be stochastically ordered in a failure rate sense by extending the above Lemma to the following corollary.

\section{Corollary 1}

Suppose $X_{i} \leq_{f} X_{j} \leq_{f} X_{k}$. Then $X_{i} \prec X_{k}$.

As a consequence of Lemma 1 and Lemma 2, for any non-negative due date, the optimal sequence is easily obtained if the processing times are stochastically ordered in a failure rate sense.

\section{Theorem 1}

If the processing times, $X_{i}, i=1, \cdots, n$, are stochastically ordered in a failure rate sense in such a way that $X_{1} \leq_{f} X_{2} \leq_{f} \cdots \leq_{f} X_{n}$, then the optimal policy processes jobs in an increasing order of index for any common due date.

Lemma 1 can be extended to problems with multiple common due date. Suppose there are multiple classes of jobs. All the jobs in the same class share their own common due date. Then partial sequence for each class of jobs can be obtained. These partial sequences are exactly the same as those obtained by applying pairwise rank based policy. Any two jobs in the same class are called class-wise adjacent if there are no other jobs in between.

\section{Lemma 3}

Consider any two class-wise adjacent jobs $i$ and $j$. If $X_{i} \prec X_{j}$, then job $i$ should precede job $j$ in an optimal sequence.

\section{Proof}

Consider a realization $\varpi \in \Omega$ of processing times. We denote the realization of $X_{i}$ by $X_{i}(\varpi)$. Also, let $S$ be the total processing time of any jobs between jobs $i$ and $j, S(\varpi)$ be its realization, and $D$ be the common due date for jobs $i$ and $j$. If $X_{i}(\varpi)<X_{j}(\varpi)$, it is better to process job $i$ first for any realization of due date $D(\varpi)$.

\subsubsection{Pairwise Rank Based Policy}

In many cases, stochastic ordering generates partial sequences only. This is from the fact that stochastic ordering requires a strong condition to be satisfied. The more likely ordering can generate partial sequences as well because the transitivity does not hold for some sets 
of random variables. However, using the relation $X_{i} \prec X_{j}$, each and every job can be compared to every other, thus it can be ranked based on how many jobs should precede it via Lemma 1 or Lemma 3. The rank of job $i$ is defined as the number of jobs that should precede job $i$. This policy of processing jobs in increasing order of rank is called 'pairwise rank based policy.'

\subsection{Probabilistic Slack Policy}

In the previous subsection, the more likely smaller relation of any two random variables, $X_{i} \prec X_{j}$, was introduced. One drawback of the relation is that it is not transitive for some random variables. Thus pairwise rank based policy is merely a heuristic. In this subsection, the probability that the processing time of job $i$ is less than or equal to the due date, i.e., $\operatorname{Pr}\left\{X_{i} \leq D\right\}$, will be discussed. The probability $\operatorname{Pr}\left\{X_{i} \leq D\right\}$ is called the probabilistic slack for job $i$. Intuition says that a sequence in which all the jobs are ordered in decreasing order of probabilistic slack might be optimal. This policy of sequencing jobs in decreasing order of probabilistic slack is called 'probabilistic slack policy.'

When probabilistic slacks tie, the shortest processing time rule gives optimal sequence that may not be obtained by probabilistic slack policy. The ties in probabilistic slacks occur, for example, when the processing times and common due date are deterministic. This is because $\operatorname{Pr}\left\{X_{i} \leq D\right\}=1$ for the jobs whose processing times are smaller than the due date.

A policy, called normalized slack policy, and the concept of myopic optimality are introduced (Jang, 2002). Job $i$ is said to be myopically better than job $j$ if it is better to process job $i$ first between the two. Job $i$ is said to be myopically optimal if no other jobs are myopically better than job $i$.

Let $E[\pi]$ be the expected number of early jobs for sequence $\pi$. By definition, job $i$ is myopically better than job $j$ if $E[\pi(i, j, \cdots)]>E\left[\pi^{\prime}(j, i, \cdots)\right]$. This condition leads to $\operatorname{Pr}\left\{X_{i} \leq D\right\}>\operatorname{Pr}\left\{X_{j} \leq D\right\}$. Since probabilistic slack policy sequences jobs in decreasing order of probabilistic slack, the following lemma is obvious.

\section{Lemma 4}

Probabilistic slack policy is myopically optimal for any processing times and common due date if there are no ties in probabilistic slacks.

Problems in which the processing times follow normal distributions and the common due date is deterministic are considered. The normalized slack of job $i$ is defined as

$$
\frac{d-\mu_{i}}{\sigma_{i}}
$$

where $d$ is the deterministic common due date. Normalized slack policy sequences jobs in decreasing order of normalized slack. This policy can be considered as a special case of probabilistic slack policy since normalized slack policy sequences jobs in non-decreasing order of normalized slack.

Problems in which the processing times are arbitrary and the common due date follows an exponential distribution are considered (Boxma and Forst, 1986; Cai and Zhou, 1999; and De et al., 1991). It is shown to be optimal to process jobs in non-increasing order of $E\left[e^{-\delta X_{i}}\right]$, where $\delta$ is the rate of the exponential random variable $D$ which the common due date follows. Since the Laplace-Stieltjes transform of $X_{i}$ at $\delta$, i.e., $E\left[e^{-\delta X_{i}}\right]$, is $\operatorname{Pr}\left\{X_{i} \leq D\right\}$, this policy is the same as probabilistic slack policy.

When processing times can be stochastically ordered, probabilistic slack policy is optimal for any common due date. Indeed, $X_{i} \leq_{s t} X_{j}$ implies $\operatorname{Pr}\left\{X_{i} \leq D\right\}$ $\geq \operatorname{Pr}\left\{X_{j} \leq D\right\}$ since the former implies $E\left[h\left(X_{i}\right)\right] \leq E$ $\left[h\left(X_{j}\right)\right]$ for any function $h(t)$ which is increasing in $t$.

$$
\begin{aligned}
& X_{i} \leq_{s t} X_{j} \\
& \Rightarrow \int_{0}^{\infty} F_{D}(t) f_{i}(t) d t \leq \int_{0}^{\infty} F_{D}(t) f_{j}(t) d t \\
& \Rightarrow \quad \operatorname{Pr}\left\{X_{i}>D\right\} \leq \operatorname{Pr}\left\{X_{j}>D\right\} \\
& \Rightarrow \quad \operatorname{Pr}\left\{X_{i} \leq D\right\} \geq \operatorname{Pr}\left\{X_{j} \leq D\right\},
\end{aligned}
$$

where $F_{D}(t)$ is the distribution function of $D$.

\section{Theorem 2}

Assume that processing times, $X_{i}, i=1, \cdots, n$, are exponentially distributed and strictly stochastically ordered in such a way that $X_{1}<_{s t} X_{2}<_{s t} \cdots<_{s t} X_{n}$ while the common due date $D$ is any non-negative random variable. In this case, probabilistic slack policy is optimal. In addition, this policy sequences jobs in increasing order of index.

\section{Proof}

Consider a sequence $\pi=\left(\pi_{1}, j, i, \pi_{2}\right)$, where $X_{i} \leq_{s t} X_{j}$. We can obtain another sequence $\pi^{\prime}$ from sequence $\pi$ by interchanging the two jobs $i$ and $j$. The difference in the expected number of early jobs between these two sequences $\pi$ and $\pi^{\prime}$ is $E[\pi]-E\left[\pi^{\prime}\right]=\operatorname{Pr}\left\{C_{\pi_{1}}+X_{j} \leq D\right\}-$ $\operatorname{Pr}\left\{C_{\pi_{1}}+X_{i} \leq D\right\}$, where $C_{\pi_{1}}$ is the completion time of partial sequence $\pi_{1}$. By conditioning on $C_{\pi_{1}}$, the difference becomes

$$
\begin{gathered}
\int_{0}^{\infty} \int_{0}^{\infty}\left(1-F_{D}(t+s)\right) f_{j}(t) d t f_{C}(s) d s \\
-\int_{0}^{\infty} \int_{0}^{\infty}\left(1-F_{D}(t+s)\right) f_{i}(t) d t f_{C}(s) d s \\
=\int_{0}^{\infty} \int_{0}^{\infty} F_{D}(t+s) f_{i}(t) d t f_{C}(s) d s \\
-\int_{0}^{\infty} \int_{0}^{\infty} F_{D}(t+s) f_{j}(t) d t f_{C}(s) d s
\end{gathered}
$$

where $f_{C}(t)$ is the probability density function of $C_{\pi_{1}}$. 
Since $F_{D}(t+s)$ is increasing in $t$ and $X_{i} \leq_{s t} X_{j}$, the last quantity is less than 0 . Therefore, we should interchange the two jobs $i$ and $j$ to maximize the expected number of early jobs. Applying the same procedure to all the remaining adjacent jobs in the sequence produces a new sequence which is stochastically ordered and maximizes the expected number of early jobs.

When processing times are not stochastically orderable, probabilistic slack policy can be optimal depending on the characteristic of the common due date. Let a random variable $D_{t}$ denote the remaining time after $t>0$ time units have passed. If $\operatorname{Pr}\left\{X_{i} \leq D_{t}\right\}>\operatorname{Pr}\left\{X_{j} \leq\right.$ $\left.D_{t}\right\}$ for all $t \geq 0$ and all $i$ and $j, i \neq j$, probabilistic slack policy sequences jobs in such a way that the expected number of early jobs is maximized.

\section{Lemma 5}

If $\operatorname{Pr}\left\{X_{i} \leq D_{t}\right\}>\operatorname{Pr}\left\{X_{j} \leq D_{t}\right\}$ for all $t \geq 0$ and all $i$ and $j$, $i \neq j$, probabilistic slack policy is optimal.

\section{Proof}

Consider a sequence $\pi=\left(\pi_{1}, j, i, \pi_{2}\right)$, where $\operatorname{Pr}\left\{X_{i} \leq\right.$ $D_{t}>\operatorname{Pr}\left\{X_{j} \leq D_{t}\right\}$. We obtain another sequence $\pi^{\prime}$ from sequence $\pi$ by interchanging the two jobs $i$ and $j$. The difference in the expected number of early jobs between these two sequences $\pi$ and $\pi^{\prime}$ is $E[\pi]-E\left[\pi^{\prime}\right]=\operatorname{Pr}\left\{C_{\pi_{1}}+\right.$ $X_{i} \leq D-\operatorname{Pr}\left\{C_{\pi_{1}}+X_{i} \leq D\right\}$, where $C_{\pi_{1}}$ is the completion time of partial sequence $\pi_{1}$. Since $\operatorname{Pr}\left\{X_{i} \leq D_{t}\right\}>\operatorname{Pr}\left\{X_{j}\right.$ $\left.\leq D_{t}\right\}$ for all $t>0$, the difference is less than 0 for any realization of $C_{\pi_{1}}$. Therefore, it is better to interchange the two jobs $i$ and $j$.

A good example of the due date $D$ that satisfies the condition of Lemma 5 is when the due date $D$ follows an exponential distribution.

\subsection{Proposed Policy}

Previously, pairwise rank based policy and probabilistic slack policy are introduced. Each policy has both advantages and disadvantages over the other. For example, pairwise rank based policy generates optimal solutions when the processing times and due date are deterministic while probabilistic slack policy generates optimal solutions whenever the common due date follows an exponential distribution.

However, probabilistic slack policy has difficulty in generating proper sequences when there are ties in probabilistic slacks. If there is a means to break the ties, the performance of probabilistic slack policy will get better.

From the sample path analysis shown in Lemma 1, it is true that if $X_{i} \prec X_{j}$, then $\operatorname{Pr}\left\{X_{i}+C \leq D\right\} \geq \operatorname{Pr}\left\{X_{j}+\right.$ $C \leq D\}$ for any non-negative random variable $C$ and common due date $D$. Conversely, it is true as well that if $\operatorname{Pr}\left\{X_{i}+C \leq D\right\}<\operatorname{Pr}\left\{X_{j}+C \leq D\right\}$, then $X_{j} \prec X_{i}$.

From the discussions so far, it can be concluded that the more likely smaller relation is a good way to break the ties in probabilistic slack policy. Therefore a new policy should sequence jobs in non-increasing order of probabilistic slacks and break ties with the more likely smaller relation.

Thus this policy is optimal even when the processing times and common due date are deterministic or nonoverlapping. A set of distributions is defined to be nonoverlapping if $\operatorname{Pr}\left\{X_{i} \leq X_{j}\right\}=0$ or 1 for all $i$ and $j, i \neq j$ (Pinedo, 1983).

\section{PROBLEMS}

\subsection{Erlang Processing Times}

For any set of the processing times which follow Erlang distributions, the transitivity for pairwise rank based policy and stochastic ordering for probabilistic slack policy are satisfied for a special case as given in the following two propositions.

\section{Proposition 1}

Suppose $X_{i} \sim \operatorname{Erlang}\left(k_{i}, \lambda_{i}\right)$ and $X_{j} \sim \operatorname{Erlang}\left(k_{j}, \lambda_{j}\right)$, where $k_{i}$ is the number of phase and $\lambda_{i}$ is the rate of each phase. If $k_{i} \leq k_{j}$ and $\lambda_{i} \geq \lambda_{j}$, then $X_{i} \prec X_{j}$.

\section{Proof}

First, we obtain $\operatorname{Pr}\left\{X_{i} \leq X_{j}\right\}$ as follows.

$$
\begin{aligned}
\operatorname{Pr}\left\{X_{i} \leq X_{j}\right\} & =\int_{0}^{\infty}\left(1-\sum_{r=0}^{k_{i}-1} \frac{\left(\lambda_{i} t\right)^{r} e^{-\lambda_{i} t}}{r !}\right) \frac{\lambda_{j}^{k_{j}} t^{k_{j}-1} e^{-\lambda_{j} t}}{\left(k_{j}-1\right) !} d t \\
& =1-\int_{0}^{\infty} \sum_{r=0}^{k_{i}-1} \frac{\left(\lambda_{i} t\right)^{r} e^{-\lambda_{i} t}}{r !} \frac{\lambda_{j}^{k_{j}} t^{k_{j}-1} e^{-\lambda_{j} t}}{\left(k_{j}-1\right) !} d t \\
& =1-\sum_{r=0}^{k_{i}-1} \int_{0}^{\infty} \frac{\lambda_{i}^{r} \lambda_{j}^{k_{j}}}{r !\left(k_{j}-1\right) !} e^{-\left(\lambda_{i}+\lambda_{j}\right) t} t^{r+k_{j}-1} d t \\
& =1-\sum_{r=0}^{k_{i}-1} \frac{\left(r+k_{j}-1\right) !}{r !\left(k_{j}-1\right) !} \frac{\lambda_{i}^{r} \lambda_{j}^{k_{j}}}{\left(\lambda_{i}+\lambda_{j}\right)^{r+k_{j}}} .
\end{aligned}
$$

If we let $\lambda_{i} /\left(\lambda_{i}+\lambda_{j}\right)=p \geq 0.5$, then the last term in the above equation is decreasing in $p \geq 0.5$ since

$$
\begin{aligned}
& \frac{d}{d p} \sum_{r=0}^{k_{i}-1} \frac{\left(r+k_{j}-1\right) !}{r !\left(k_{j}-1\right) !} p^{r}(1-p)^{r+k_{j}} \\
& \quad=\sum_{r=0}^{k_{i}-1} \frac{\left(r+k_{j}-1\right) !}{r !\left(k_{j}-1\right) !}(1-p)^{k_{j}-1} p^{r-1}\left(r(1-p)-k_{j} p\right) \\
& \quad<0 .
\end{aligned}
$$

From the fact that $\operatorname{Pr}\left\{X_{i} \leq X_{j}\right\}=0.5$, we have $\sum_{r=0}^{k_{i}-1}$ $\frac{\left(r+k_{j}-1\right) !}{r !\left(k_{j}-1\right) !} 0.5^{r} 0.5^{r+k_{j}}=0.5$. Therefore we have 


$$
\begin{aligned}
& \sum_{r=0}^{k_{i}-1} \frac{\left(r+k_{j}-1\right) !}{r !\left(k_{j}-1\right) !} \frac{\lambda_{i}^{r} \lambda_{j}^{k_{j}}}{\left(\lambda_{i}+\lambda_{j}\right)^{r+k_{j}}} \\
& \leq \sum_{r=0}^{k_{j}-1} \frac{\left(r+k_{j}-1\right) !}{r !\left(k_{j}-1\right) !} \frac{\lambda_{i}^{r} \lambda_{j}^{k_{j}}}{\left(\lambda_{i}+\lambda_{j}\right)^{r+k_{j}}}
\end{aligned}
$$

The above equation implies $\operatorname{Pr}\left\{X_{i} \leq X_{j}\right\} \geq 0.5$.

\section{Proposition 2}

Suppose $X_{i} \sim \operatorname{Erlang}\left(k_{i}, \lambda_{i}\right)$ and $X_{j} \sim \operatorname{Erlang}\left(k_{j}, \lambda_{j}\right)$, where $k_{i}$ is the number of phase and $\lambda_{i}$ is the rate of each phase. If $k_{i} \leq k_{j}$ and $\lambda_{i} \geq \lambda_{j}$, then $X_{i} \leq_{s t} X_{j}$.

\section{Proof}

If $X \sim \operatorname{Erlang}(k, \lambda)$, then its distribution function can be represented by $F(x)=1-\sum_{r=0}^{k-1} \frac{(\lambda x)^{r}}{r !} e^{-\lambda x}$. From the following,

$$
\begin{aligned}
\frac{d}{d \lambda} \sum_{r=0}^{k-1} \frac{(\lambda x)^{r}}{r !} e^{-\lambda x} & \\
& =-x e^{-\lambda x}+\sum_{r=0}^{k-1} \frac{d}{d \lambda}\left(\frac{(\lambda x)^{r}}{r !} e^{-\lambda x}\right) \\
& =-x e^{-\lambda x}+\sum_{r=0}^{k-1} x e^{-\lambda x}\left(\frac{(\lambda x)^{r-1}}{(r-1) !}-\frac{(\lambda x)^{r}}{r !}\right) \\
& =x e^{-\lambda x}\left(\sum_{r=0}^{k-2} \frac{(\lambda x)^{r}}{r !}-\sum_{r=0}^{k-1} \frac{(\lambda x)^{r}}{r !}\right) \\
& =-x e^{-\lambda x} \frac{(\lambda x)^{k-1}}{(k-1) !} \\
& <0,
\end{aligned}
$$

it is noted that $F(x)$ is increasing in $\lambda$. Therefore, it is obvious that $F(x)$ is decreasing in $k$. Thus we have

$$
\begin{aligned}
& 1-\sum_{r=0}^{k_{i}-1} \frac{\left(\lambda_{i} x\right)^{r}}{r !} e^{-\lambda_{i} x} \\
& \geq 1-\sum_{r=0}^{k_{i}-1} \frac{\left(\lambda_{j} x\right)^{r}}{r !} e^{-\lambda_{j} x} \\
& \geq 1-\sum_{r=0}^{k_{j}-1} \frac{\left(\lambda_{j} x\right)^{r}}{r !} e^{-\lambda_{j} x},
\end{aligned}
$$

which leads to $F_{i}(x) \geq F_{j}(x)$ for all $x$, where $F_{i}(x)$ and $F_{j}(x)$ are the distribution functions of $X_{i}$ and $X_{j}$, respectively.

If only a subset of processing times can be stochastically ordered, both pairwise rank based policy and probabilistic slack policy cannot always generate optimal sequences. However, these two policies are believed to be quite good heuristics when the processing times and common due date follow arbitrary distributions.

\subsection{Exponential Due Dates}

A good example of exchangeable due dates is the case where all the due dates are independently and identically distributed. Whereas there is only one realization of common due date, there might be many different realizations for exchangeable due dates.

The following theorem is proved for cases where processing times can be stochastically ordered in stochastic ordering sense (Boxma and Forst, 1986).

\section{Theorem 3}

Assume that processing times, $X_{i}, i=1, \cdots, n$, are stochastically ordered in such a way that $X_{1} \leq_{s t} X_{2} \leq_{s t} \cdots \leq_{s t}$ $X_{n}$ and all the due dates are any i.i.d. non-negative random variables. The optimal policy for maximizing the expected number of early jobs processes jobs in increasing order of index.

Theorem 3 implies that probabilistic slack policy is optimal when processing times can be stochastically ordered and due dates are exchangeable. Similarly, pairwise rank based policy is also applicable when due dates are exchangeable.

\section{Lemma 6}

Consider any two adjacent jobs $i$ and $j$, in a sequence. If $X_{i} \prec X_{i}$, optimal policy should sequence job $i$ before job $j$ for any exchangeable due dates.

\section{Proof}

Consider a realization, $\varpi \in \Omega$, of processing times. In addition, denote the realization of $X_{i}$ by $X_{i}(\varpi)$. When $X_{i}(\varpi)<X_{i}(\varpi)$, there are three cases where processing job $i$ first is worse than the other way around. Those cases are cancelled out since due dates are exchangeable. For example (see Figure 1), the probability that due dates for jobs $i$ and $j$ occurs in Zone I and Zone IV, respectively, is the same as the probability that due dates for jobs $i$ and $j$ occurs in Zone IV and Zone I, respectively. Consequently, given a realization of the processing times $\varpi$, the expected reward becomes larger when the shorter job is processed first. Therefore, with probability $\operatorname{Pr}\left\{X_{i} \prec X_{j}\right\}$, it is better to process job $i$ first.

As long as the transitivity holds for processing times, pairwise rank based policy is guaranteed to be optimal for any exchangeable due dates. The new policy can be used in case of exchangeable due dates as well.

Just like the common due date case, multiple classes of exchangeable due dates can be considered in which a set of jobs within a class share any i.i.d. due dates. If any two jobs $i$ and $j$ are classwise-adjacent and $X_{i} \prec X_{j}$, then optimal policy should sequence job $i$ before job $j$ as given in the following lemma. 


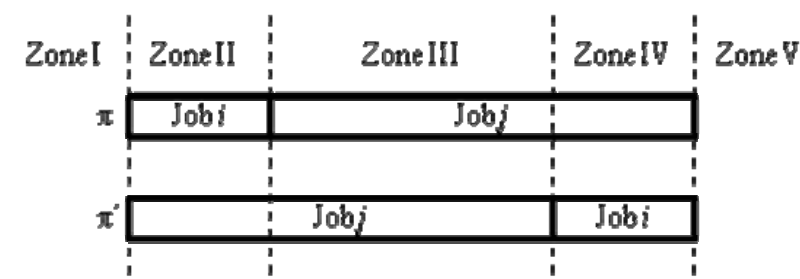

\begin{tabular}{|c|c|c|}
\hline \multicolumn{2}{|c|}{ Due Date Occurrence } & \multirow{2}{*}{ Difference } \\
\cline { 1 - 2 } Job $i$ & Job $j$ & 0 \\
Zone I & Zone I & 0 \\
Zone I & Zone II & 0 \\
Zone I & Zone III & -1 \\
Zone I & Zone IV & 0 \\
Zone I & Zone V & 0 \\
\hline Zone II & Zone I & 0 \\
Zone II & Zone II & 0 \\
Zone II & Zone III & -1 \\
Zone II & Zone IV & 0 \\
Zone II & Zone V & 1 \\
Zone III & Zone I & 1 \\
Zone III & Zone II & 1 \\
Zone III & Zone III & 0 \\
Zone III & Zone IV & 1 \\
Zone III & Zone V & 1 \\
\hline Zone IV & Zone I & 1 \\
Zone IV & Zone II & 1 \\
Zone IV & Zone III & 0 \\
Zone IV & Zone IV & 1 \\
Zone IV & Zone V & 0 \\
\hline Zone V & Zone I & 0 \\
Zone V & Zone II & 0 \\
Zone V & Zone III & -1 \\
Zone V & Zone IV & 0 \\
Zone V & Zone V & \\
\hline
\end{tabular}

Figure 1. Illustration for the Proof of Lemma 6

\section{Lemma 7}

Consider any two class-wise adjacent jobs $i$ and $j$. If $X_{i} \prec X_{j}$, then optimal policy should sequence job $i$ before job $j$.

\section{Proof}

The proof is similar to that of Lemma 6. See Figure 2.

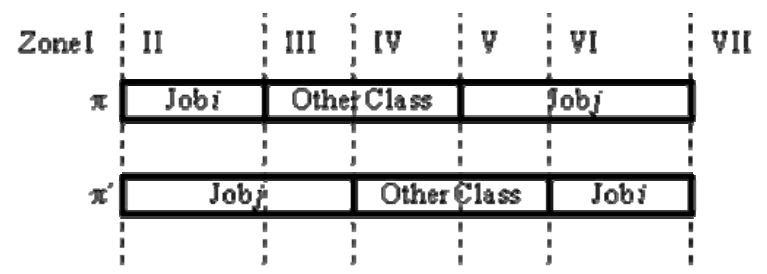

Figure 2. Illustration for the Proof of Lemma 7

\section{FINAL REMARKS}

In this paper, stochastic scheduling problems with a common due date and exchangeable due dates are considered. Based on a sample path analysis, pairwise rank based policy which orders more likely smaller jobs first has been introduced. It is shown that pairwise rank based policy is optimal when processing times are stochastically ordered in failure rate sense.

In addition, probabilistic slack policy has also been introduced. It is shown that when either processing times are stochastically ordered or due date is exponential, probabilistic slack policy is optimal.

It is shown that both policies are complementary to each other, for example, if processing times and due date are deterministic, pairwise rank based policy is optimal, but probabilistic slack policy is not guaranteed to be optimal. On the other hand, as long as due date is exponential, probabilistic slack policy is optimal, but pairwise rank based policy is not always.

The proposed policy captures advantages of both policies. This policy sequences jobs in non-increasing order of probabilistic slacks and break ties by the more likely smaller relation. The new policy is optimal when either pairwise rank based policy or probabilistic slack policy is optimal

\section{REFERENCES}

Boxma, O. J. and F. G. Forst, "Minimizing the Expected Weighted Number of Tardy Jobs in Stochastic Flow Shops," Operations Research Letters 5 (1986), 119126.

Cai, X. and S. Zhou, "Stochastic Scheduling on Parallel Machines subject to Random Breakdowns to Minimize Expected Costs for Earliness and Tardy Jobs," Operations Research 47 (1999), 422-437.

Choi, J. and H. Kim, "A Review on Scheduling Problems for Minimizing the Number of Late Jobs," Korean Production and Operations Management Society 22 (2011), 159-175.

De, P., J. B. Ghosh, and C. E. Wells, "On the Minimization of the Weighted Number of Tardy Jobs with Random Processing Times and Deadline," Computers and Operations Research 18 (1991), 457463.

Jang, W., "Dynamic Scheduling of Stochastic Jobs on a Single Machine," European Journal of Operational Research 138 (2002), 518-530.

Li, W. and K. D. Galzebrook, "On Stochastic Machine Scheduling with General Distributional Assumptions," European Journal of Operational Research 105 (1998), 525-536.

Pinedo, M., "Stochastic Scheduling with Release Dates and Due Dates," Operations Research 31 (1983), 559-572.

Sarin, S. C., E. Erel, and G. Steiner, "Sequencing Jobs on a Single Machine with a Common Due Date and Stochastic Processing Times," European Journal of Operational Research 51 (1991), 188-198.

Ross, S. M., "Stochastic Processes," John Wiley and Sons, New York, 1996.

Sterna, M., "Late work scheduling in shop systems," Publishing House of Poznan University of Technology, Poznan, Polland, 2006. 